\title{
The effectiveness of exercise intervention for academic achievement, cognitive function, and physical health among children in Mongolia: a cluster RCT study protocol
}

Kenji Takehara', Togoobaatar Ganchimeg², Akihito Kikuchi', Lkagvasuren Gundegmaa ${ }^{5}$, Lkagvasuren Altantsetseg ${ }^{5}$, Ai Aoki ${ }^{1,6}$, Takemune Fukuie $^{3}$, Kazuya Suwabe ${ }^{3,4}$, Shagdar Bat-Erdene ${ }^{5}$, Masashi Mikami ${ }^{7}$, Rintaro Mori ${ }^{1 *}$ and Hideaki Soya ${ }^{3,4}$

\begin{abstract}
Background: Many studies have demonstrated positive effects of physical activity on children's health such as improved cardiorespiratory function and decreased obesity. Physical activity has also been found to have positive effects on academic achievement and cognitive function. However, there are few high quality RCT studies on this topic at present and the findings remain controversial.
\end{abstract}

Methods: This protocol describes cluster randomized controlled trials assessing the impact of school-based exercise intervention among children in Mongolia. The intervention consists of 3-min sessions of high intensity interval training combined with music implemented two times a week at school during study periods. The participants are children in the fourth grade in public elementary schools in the Sukhbaatar district in Ulaanbaatar, Mongolia. The participants are cluster randomized by school and allocated either to the intervention or control group. The primary outcome is academic achievement. Secondary outcomes are obesity/overweight, physical fitness function, lifestyle, mental health, and cognitive function.

Discussion: This cluster-RCT is designed and implemented to assess the effectiveness of exercise intervention on academic achievement, cognitive function, and physical and mental health among school-age children in Mongolia. This study will provide evidence to promote physical activities among children in low- and middle- income countries.

Trial registration: UMIN: UMIN000031062. Registered on 1st February 2018.

Keywords: RCT, Physical activity, Exercise, Academic achievement, Physical fitness, Cognitive function, Children

\footnotetext{
* Correspondence: rintaromori@gmail.com

${ }^{1}$ Department of Health Policy, National Center for Child Health and

Development, 2-10-1, Okura, Setagaya, Tokyo 157-8535, Japan

Full list of author information is available at the end of the article
}

(c) The Author(s). 2019 Open Access This article is distributed under the terms of the Creative Commons Attribution 4.0 International License (http://creativecommons.org/licenses/by/4.0/), which permits unrestricted use, distribution, and reproduction in any medium, provided you give appropriate credit to the original author(s) and the source, provide a link to the Creative Commons license, and indicate if changes were made. The Creative Commons Public Domain Dedication waiver (http://creativecommons.org/publicdomain/zero/1.0/) applies to the data made available in this article, unless otherwise stated. 


\section{Background}

Physical inactivity is becoming an urgent health issue. Although physical inactivity is highest in developed countries, it is also on the rise among middle-income earners in developing countries including their children [1]; the WHO reported that $81 \%$ of adolescents aged $11-17$ years worldwide had insufficient levels of physical activity. [1]

Previous studies demonstrated a strong relationship between physical inactivity and non-communicable diseases such as obesity, hypertension, diabetes, elevated blood lipids, heart attack, heart failure, stroke, and breast and colon cancers $[1,2]$. Physical activity in childhood is crucial for the healthy development of musculoskeletal tissue, the cardiovascular system, neuromuscular awareness, and prevention of obesity and diabetes. [1,3] Moreover, recent studies have suggested that physical activity contributes to improving brain function and mental health. [4-8] Several ongoing studies are focusing on the positive effects of physical activity in broader areas such as academic achievement, cognitive function, mental health, and social skills. [3, 6, 9-15]

Academic achievement is a composite measure of many different factors from the developmental to the psychosocial. Several mechanisms thought to be instrumental in improving academic achievement have been reported from the neurobiological, psychological, and physical perspectives. Neurobiologically, exercise induces increased cerebral blood flow, brain-derived neurotrophic factors, neuroplasticity, and changes in neurotransmitters such as serotonin, noradrenalin, and dopamine. $[4,5]$ For example, the acquisition of motor skills was shown to improve neuroplasticity and cognitive function, [5] and improvements in executive function, memory, and attention have been reported in connection with increased physical activity. [16-20] The executive function is considered to be especially important for predicting children's academic achievement. [21] Psychologically, exercise is thought to improve mood, lessen anxiety, and boost self-perception/self-esteem, all of which contribute to better academic achievement. [3, 11, 22] Numerous studies have demonstrated the positive effects of exercise on academic achievement and cognitive function, but most were small randomized controlled trials (RCTs), and some were not intervention studies. [10, 16, 17, 23-26] To date there are very few, large, population-based RCT examining the effect of exercise in children. A small number of meta-analyses and systematic reviews have been done but have demonstrated only small positive effects due to exercise intervention. $[8,12,27]$ A Cochrane systematic review recommended investigating academic achievement and brain function in obesity-prevention studies because only few RCT addressing these issue exist [28] and the current evidence is meager. High-quality RCT are needed especially in developing countries as most of the previous intervention studies were done in developed countries like the US, Canada, and Australia.
As studies of children in the developed world have shown, physical activity deficits among children not only result in increased obesity and poorer athletic ability but may also have adverse effects on academic achievement and brain function. Similar trends are now being seen among children in emerging nations. Mongolia is one of these emerging nations now facing an epidemiological transition from infectious diseases to non-communicable and lifestyle-related health issues. In Mongolia, the physical activity deficit among children has become a serious problem in recent years especially in Ulaanbaatar, the capital city, where the shortage of outdoor play spaces caused by rapid urbanization, the spread of television and video games, and severe winter weather, have reduced the physical activity levels of children. In Ulaanbaatar, the average temperature is below freezing for more than five months per year. This fact has contributed greatly to increasing child obesity. In Mongolia, previous studies showed that the rate of overweight and obesity was 10.6 and $1.5 \%$, respectively, among children aged 12-17. [29] Physical inactivity and obesity cause childhood health problems including diabetes mellitus, hypertension, hyperlipidemia, etc. Moreover, the adverse effects of exercise deficits are thought to have non-physical consequences including poor academic achievement and cognitive development. These health problems are common to middle-income earners worldwide, including those in developing countries like Mongolia.

The primary objective of our study is to investigate the effectiveness of exercise intervention on academic achievement among children using a large cluster RCT. The secondary objective is to investigate the effectiveness of exercise intervention on reducing the prevalence of obesity and overweight and on improving physical fitness performance, lifestyle, mental health, and cognitive function. The executive function was chosen as the target cognitive function based on the hypothesis that this function can best predict academic achievement.

\section{Methods/design}

This study will be a collaborative effort by the National Center for Child Health and Development, Mongolian National Institute of Physical Education (MNIPE), and the University of Tsukuba. This study is a cluster-RCT using school as a cluster. A pilot study has already been done to confirm the feasibility and logistics of the planned intervention program and the assessment of the subjects' physical fitness and cognitive function at one school in the study area.

\section{The study setting}

This study will be conducted in the Sukhbaatar district of Ulaanbaatar, Mongolia and will recruit 10 public schools out of 11 public schools in the district. The 
population of this district in 2017 was 136,569 or roughly $10 \%$ of the population of Ulaanbaatar. [30]

One school which is not recruited for this study participated in the pilot study. Ten schools will later be allocated to the intervention or control group. Six, three, and two schools are located in urban or residential, ger (Mongolian traditional dwelling), and mixed residential areas, respectively. Each school has a total of 108 to 307 children in four to nine fourth-grade classes.

\section{Participants}

Participants are children in the fourth grade in public elementary schools in the study area. The inclusion criteria are: [1] attendance at a public school in the Sukhbaatar district; [2] written consent from parents or guardians; and [3] age-appropriate literacy in Mongolian. The exclusion criteria are: [1] comorbidities or contraindications prohibiting participation in an exercise program and [2] enrollment in a special needs program.

\section{Intervention}

The exercise program for the intervention group will be implemented two times per week at school in twiceweekly physical education classes required by the educational curriculum. The exercise program combines high intensity interval training and music. The exercise program will consist of four segments with different target activities and short rest intervals between segment. The target activities of each segment are warm-up, agility and high-speed movements, side-steps, and jumps. The program requires $3 \mathrm{~min}$. In the first phase of intervention, the participants will practice each segment one by one to synchronize their movements with the music. Including stretching and explanation of the program before the exercise program, it will take about $20 \mathrm{~min}$. The first phase will last for six weeks. Subsequently, in the second phase of the intervention, the participants will perform all the segments sequentially. Including stretching, it will take 10 min. The second phase will last for four weeks.

The program, based on high intensity interval training (HIIT), will consist of high intensity phases involving steps, jumps, and squats alternating with intervals of rest. HIIT has been found to improve physical endurance effectively. Some studies using small samples have also reported that HIIT improved not only physical endurance but also cognitive function more efficiently to compare with moderate-intensity endurance training. [31-34] This exercise program uses music, sound effects and verbal instructions in Mongolian to help children, especially those who are poor at physical activities, to acquire the movement easily. For this purpose, the tempo of music is set at 105-140 bpm. The music is also chosen carefully to amuse and motivate children. $[35,36]$ This program does not use self-paced or acquired movements such as walking. Through this program, the participants will aim to acquire a variety of motor skills, which is thought to correlate with improvements in cognitive function. This intervention will be administered by university students at MNIPE working as research assistants (RA). The RA will undergo a five-day course covering the aims of the program, appropriate practices, safety measures including ensuring the health and well-being of the participants, and training in implementing the exercise regimen. The RA will be instructed using standardized materials at the course and receive a few days additional course to improve the quality of intervention program during the intervention period. This program will be implemented in a space available at each school, such as the gymnasium or conference room. Although practice is recommended at least two times per week, each school will decide the actual frequency. There are no criteria for discontinuing participation or modifying the allocation. During the study period, MNIPE researchers will visit schools in the intervention group on a regular basis and supervise implementation of the program. In the control arm, the participants will attend the usual physical education class only.

\section{Primary outcome}

The primary outcome of academic achievement will be assessed by the participants' performance on the examination in 2018, which consists of a mathematics and Mongolian language portion. The participants' score on the test for May 2017 at the end of third grade will be used as the baseline data.

\section{Secondary outcomes}

The proportion of obesity and overweight, and the physical fitness function, lifestyle, and mental health, and cognitive function measures between November and December 2018 will be the secondary outcomes. All measures will be obtained in between February and April in 2018 as baseline data.

The researchers and RAs will obtain physical measurements (height and body weight) and physical fitness function test data to establish the baseline in February and at the end of study. The body mass index (BMI) will be calculated, and the proportion of participants classified as obese or overweight using the WHO/CDC criteria will be calculated. Physical fitness function will be assessed by participants' performance on a $20-\mathrm{m}$ shuttle run and side-to-side jumps.

Participants' parents or guardians will answer a selfadministered questionnaire on lifestyle covering their children's sleeping hours, exercise habits, time spent viewing television or using video games, children's household tasks, and their mental and physical health. Children's physical health condition will be assessed using a five-point Likert 
scale. The Strengths and Difficulties Questionnaire (SDQ), which has already translated into Mongolian and backtranslated, [37] will be used to assess the mental health of the participants. The cut-off values on the SDQ published in the literature and available on the official website for 'normal,' 'borderline', and 'abnormal' will be adopted. [38]

To assess cognitive function, the Flanker test, a representative cognitive function test specifically assessing inhibitory control of the executive function, will be used. [39] The Flanker test will be administered using a specially-designed app for use on a tablet at school under the instruction of the researchers. The response time and correct rate for 50 tasks, including congruent and incongruent tasks, will be measured. Before the actual test, every student will do a practice test consisting of ten tasks once or twice.

The intervention will begin in April 2018. Randomized allocation is done in February 2018 and baseline data collection will be done from February to April 2018. The intervention period will be interrupted by summer vacation between the end of June and the beginning of September 2018. The intervention period before summer vacation is the first phase of the intervention and after summer vacation is the second phase of the intervention. Postintervention data will be collected between November and December 2018. (Fig. 1).

\section{Sample size}

Some 2445 children are enrolled in fourth grade at public elementary schools in the Sukhbaatar district of Ulaanbaatar, Mongolia. 2337 children are enrolled in the ten schools that participate in this study. All the eligible children will be recruited.

This study is a longitudinal cluster randomized trial which has three-level cluster units: the school, individual, and measurement time point. Additionally, the number of students at each school differs. To calculate the statistical power, we implemented a simulation study extending the method of a previous study to adjust for unbalanced data. [40] We assumed the standardized difference of the primary outcome to be equal to 0.2 , and the dropout rate to be equal to $5 \%$. Since the statistical power of the longitudinal cluster randomized trial depends on the correlation between measurement time points for the same individual, we assumed values of $0.4,0.5$ or 0.6 . Bonferroni correction was used to adjust for the multiplicity of statistical testing calculated for each site (Urban/ger/mixed residential). Table 1 shows the statistical power and alpha error for each site. We also simulated the disjunctive alpha error and power. In all assumption settings, the disjunctive alpha error is kept nearly equal to 0.05 , and the statistical power of the urban site exceeds 0.8 . Additionally, under conditions in which the correlation equals 0.6 , the statistical power exceeds 0.8 for both sites. The simulation was done 5000 times using the mixed procedure with SAS Software 9.4 (SAS Inc., Cary, NC, USA).

\section{Allocation}

We will perform stratified block randomization to allocate ten schools into the intervention and control arms. These schools will be stratified into the urban area group (6 schools) and the ger or mixed residential area group

\begin{tabular}{|c|c|c|c|c|c|c|c|c|c|c|c|c|c|c|c|}
\hline & & \multicolumn{14}{|c|}{ STUDY PERIOD } \\
\hline & & \begin{tabular}{|l|} 
Enrolment \\
\end{tabular} & Allocation & & & & & & -alloce & & & & & & 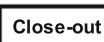 \\
\hline \multirow{2}{*}{ TIMEPOINT } & 2017 & \multicolumn{13}{|c|}{2018} & 2019 \\
\hline & May & Feb & Feb & Feb & Mar & Apr & May & Jun & Jul & Aug & Sep & Oct & Nov & Dec & Jan \\
\hline \multicolumn{16}{|l|}{ ENROLMENT: } \\
\hline Eligibility screen & & $\bar{x}$ & & & & & & & & & & & & & \\
\hline Informed consent & & $\bar{x}$ & & & & & & & & & & & & & \\
\hline Allocation & & & $x$ & & & & & & & & & & & & \\
\hline \multicolumn{16}{|l|}{ INTERVENTIONS: } \\
\hline \multicolumn{16}{|l|}{ Exercise program } \\
\hline \multicolumn{16}{|l|}{\begin{tabular}{|l|} 
ASSESSMENTS: \\
\end{tabular}} \\
\hline $\begin{array}{c}\text { Academic } \\
\text { achievement }\end{array}$ & $x$ & & & & & & & & & & & & & $x$ & \\
\hline Physical fitness & & & & $\bar{x}$ & $\mathrm{x}$ & $\bar{x}$ & & & & & & & $\bar{x}$ & $\bar{x}$ & \\
\hline \multirow{2}{*}{$\begin{array}{r}\text { Cognitive function } \\
\text { Height/ body } \\
\text { weight }\end{array}$} & & & & $x$ & $\bar{x}$ & $\bar{x}$ & & & & & & & $x$ & $\mathrm{x}$ & \\
\hline & & & & $x$ & $x$ & $x$ & & & & & & & $x$ & $x$ & \\
\hline Physical health & & & & $\bar{x}$ & & & & & & & & & & $\mathrm{x}$ & \\
\hline Mental Health & & & & $\bar{x}$ & & & & & & & & & & $\bar{x}$ & \\
\hline Life style & & & & $\mathrm{x}$ & & & & & & & & & & $x$ & \\
\hline
\end{tabular}

Fig. 1 The schedule of enrolment, interventions and assessments 
Table 1 Simulation results of statistical power

\begin{tabular}{|c|c|c|c|c|c|c|}
\hline \multirow[t]{3}{*}{ Correlation } & \multicolumn{6}{|c|}{ Disjunctive: 5000 simulations Standardized difference $=0.2$} \\
\hline & \multicolumn{3}{|c|}{ Alpha error } & \multicolumn{3}{|l|}{ Power } \\
\hline & Total & Urban & Ger or mixed residential & Total & Urban & Ger or mixed residential \\
\hline 0.4 & 0.050 & 0.027 & 0.024 & 0.957 & 0.871 & 0.642 \\
\hline 0.5 & 0.047 & 0.023 & 0.024 & 0.977 & 0.920 & 0.719 \\
\hline 0.6 & 0.047 & 0.023 & 0.024 & 0.992 & 0.967 & 0.814 \\
\hline
\end{tabular}

(4 schools) according to the location of the school. To ensure a balance between the two study arms, we will order the schools by the number of fourth grade students to form blocks for randomization in each residential group. Within the blocks, the schools will be randomly allocated to the intervention or control arm. To allocate the schools, arbitrary numbers given by the representative of each school will be used. The arbitrary numbers will be processed by a program on $\mathrm{R}$ statistical software, version 3.4.3 (The $\mathrm{R}$ foundation for statistical computing platform) which is developed by a researcher. Each school will be randomly allocated to intervention or control group based on the output of the R program.

The allocation will be conducted and announced to the representative of each school at a kickoff meeting.

\section{Blinding}

According to the nature of the intervention, the participants and outcome raters, participants' parents or guardians answering the questionnaires, and researchers taking the anthropometric and physical fitness function measurements will not be blinded to the allocation. However, the chief data analyst will be blinded to the allocation.

All data collected in this study will be entered in duplicate and managed using Microsoft Excel. The data file will be secured with a password, and access to information will be limited to designated members such as the researchers and RA. Paper-based documents such as consent forms, questionnaire response sheets, and forms for recording the physical fitness function and anthropometric measurements will be stored at a research institute in Ulaanbaatar. The data from the Flanker test will be stored on tablets and the internet server.

\section{Statistical methods}

\section{Analysis of the primary outcome}

The baseline data of the intervention and control groups will be compared. The chi-squared test will be used for binary variables and the t-test will be used for continuous variables. To avoid overestimating the impact of the intervention, an intention-to-treat approach will be adopted to evaluate the impact on the primary and secondary outcomes. In this study, an individual's data will be analyzed in the originally allocated arm even if the individual moves to a school in another area during study period.
To examine the effect of the intervention while taking the hierarchical structure into consideration, we will use a linear mixed effect model. In this model, the fixed effects are the group (intervention/control), site (Urban/ ger or mixed residential), and measurement time point with the following two interaction terms: the interaction between group and measurement time point, and the interaction between the group, measurement time point, and site. The random effects are school and individual. The degrees of freedom will be estimated using Satterthwaite approximation. For the primary analysis, we will compare the participants' performance on the national year-end examination before and after the intervention using least squares means estimated by the interaction term of the group, measurement time point, and site. The Bonferroni method will be used as a multiplicity adjustment method. A significance level of 0.025 will be set for a two-sided test, and the $95 \%$ confidence interval will be calculated.

\section{Analysis of secondary outcomes}

In the secondary analysis, the same analytical method will be used as in the primary analysis for each test score (mathematics and language) and the continuous outcomes of BMI, sleeping hours, 20m shuttle run, each element of the Flanker test, side-to-side movement, and each SDQ score for mental health. We will use mixed-effects logistic regression for binary data. The covariate of this model is also the same as in the primary analysis. Although the frequency of exercise and playing outside hours constitute ordered data, they will be converted into binary data using a cutoff in the secondary analysis. The cutoff of the frequency of exercise will be between " $1-2$ days" and " $3-4$ days", playing outside hours will be between " $30 \mathrm{~min}-59 \mathrm{~min}$ " and "60 min-119 min". Since the analysis of the secondary outcome is the exploratory evaluation, we will not adjust for the multiplicity of statistical testing.

In the analysis of cognitive function using the Flanker test, participants who fail to reach a correct rate of $50 \%$ will be excluded on the assumption that they do not understand the test.

In this study, any imputation will not be performed to missing data due to lost participants or imperfect data collection. The data will be analyzed using STATA V.13.1 (STATA/IC 13.1 for Mac, STATA Corp, Lakeway Drive 
College Station, Texas), SAS Software 9.4 (SAS Inc., Cary, NC, USA), and SPSS statistics V.19.0 (IBM SPSS Statistics for Windows, IBM Corp, Armonk, New York).

\section{Subgroup analysis}

The effect of the intervention will be further analyzed using four subgroup analyses. The first subgroup analysis will focus on the location of the schools (urban and non-urban areas), the second on gender, and the third on baseline physical fitness performance using the median score on a $20 \mathrm{~m}$ shuttle run. The fourth subgroup analysis will focus on baseline academic achievement using the total score on the national year-end examination in May 2017 and the median score. The fifth subgroup analysis will be done after excluding participants whose correct rate on the Flanker test is below $80 \%$ at either the baseline or endline surveys.

\section{Other additional analysis}

An additional analysis will be done after adjusting the sample size of each cluster to a certain number. The number will be decided according to the number of participants in the smallest cluster.

\section{Discussion}

This paper is a study protocol for a cluster-RCT to assess the effectiveness of exercise intervention on academic achievement, cognitive function, and physical and mental health among school-age children in Mongolia. The intervention program combines exercise and music and was designed for use with an educational curriculum already filled with many subjects and activities.

\section{Monitoring}

Although this study is an intervention trial, the intervention is not considered to be invasive. Thus, no data monitoring committee or auditing will be needed. To ensure compliance with the intervention program, an RA will be assigned to each primary school in the intervention arm to take charge of monitoring and will be responsible for biweekly reports on the progress of the study including the frequency of the intervention program at the respective school. Researchers both in Mongolia and Japan will discuss the progress of the study and issues to be addressed via Skype on a biweekly basis in addition to frequent e-mail communication.

\section{Risks, burdens, and benefits}

The intervention program will consist of high intensity interval training. However, the physical stresses induced by the intervention, such as an increase in respiratory rate, heart rate, and sweating, are assumed to fall within the range of normal physical activity and to require a brief recovery time, given appropriate rest and hydration. In the pilot study, we confirmed that the rating of perceived exertion (RPE) using the Borg Rating of Perceived Exertion Scale normalized after the intervention. [41] The Ethical Guidelines for Medical and Health Research Involving Human Subjects in Japan have also assessed the intervention planned for this study, which is very similar to the physical fitness function test used in Japanese schools, to be non-invasive. Based on these assessments, the exercise regimen planned for this study is not considered to be harmful to the participants.

Prior to the commencement of the study, local researchers and teachers will be instructed in appropriate and safe practices for avoiding the risk of medical emergencies during the implementation of the intervention. The burden of responding to the questionnaire will fall to the parents or guardians of the participants, while the participants will have the burden of undergoing the cognitive function test. This part of the study will provide no direct benefit to the participants.

\section{Modification and inquiry}

Any modification or inquiry regarding the study will be communicated through the Department of Health Policy at the National Center for Child Health and Development. During the study period, a research office will be opened at the MNIPE. Inquiries from Mongolia will be gathered at the research office and processed by the Department of Health Policy at the National Center for Child Health and Development.

\section{Confidentiality}

The personal data including school information, class, name, social security number, etc. contained in the consent forms will be stored at the MNIPE. All data will be anonymized using a research ID number when they are entered into a computer for storage. The research ID and corresponding table will be stored at the MNIPE.

\section{Post trial care}

If the study demonstrates no negative effects, school teachers and children in the control groups will be taught the exercise program by the RAs after the study.

\section{Dissemination policy}

The results of this study will be disseminated to professionals and organizations in education and shared publicly throughout Mongolia. The study findings will also be disseminated at peer reviewed scientific journals and presented at international conferences and conferences in Japan, Mongolia, and other countries.

\section{Abbreviations}

BMI: Body mass index; CDC: Centers for Disease Control and Prevention; HIIT: High intensity interval training; MNIPE: Mongolian National Institute for Physical Education; RA: Research assistant; RCT: Randomized controlled trial; RPE: Rating of perceived exertion; SDQ: The Strength and Difficulties

Questionnaire; WHO: World Health Organization 


\section{Acknowledgements}

The authors thank the teachers at the primary school, the parents, the children who participated in the pilot tests, and the students at MNIPE who collected data and taught the exercise program to the subjects. The authors thank Dr. Lamjav Sosorbaram for field arrangement for the study.

\section{Availability of data and material}

The datasets generated and/or analysed during the current study are not publicly available but are available from the corresponding author on reasonable request.

\section{Funding}

This work was supported by JSPS KAKENHI Grant Number $17 \mathrm{H} 04501$ (to RM), $16 \mathrm{H} 06405$ (to HS). The study protocol underwent peer review by the funding body. The funding body does not have any roles in the design of this study, data collection, data analysis, interpretation of result, or writing the manuscript.

\section{Authors' contributions}

$K T, T G, A K, L G, L A, A A, S B, M M, R M$ and $H S$ participated in the design of this study. AK developed the intervention programs including the music, sound effects, and verbal instructions. KT, TG, AK, LG, SB, AA, TF and RM conducted the pilot study. TF, KS, and HS developed the tablet applications for the Flanker test. $K T, T G, L G, L A$ and $S B$ contributed to assessing the logistics of study. MM performed the simulation of statistical power and provided input from the statistical perspective. KT and AA prepared the first draft of this manuscript. All the listed authors reviewed and approved the final manuscript for publication.

\section{Ethics approval and consent to participate}

The study protocol was approved by the Institutional Review Board and Ethics Committee of the National Center for Child Health and Development, Tokyo, Japan (Number 1541) and the Mongolian National Institute of Physical Education, Ulaanbaatar, Mongolia (Number 238).

Consent to participate in this study was obtained from the directors of the schools in the study area. As the participants are minors, the participants will be enrolled after consent from the primary guardian/ parents is obtained using a form distributed to the parents or guardians by the schools. Researchers will encourage teachers and parents or guardians to explain the study to the children, but the children's consent will not be required.

\section{Consent for publication}

\section{Not applicable.}

\section{Competing interests}

None to declare.

\section{Publisher's Note}

Springer Nature remains neutral with regard to jurisdictional claims in published maps and institutional affiliations.

\section{Author details}

'Department of Health Policy, National Center for Child Health and Development, 2-10-1, Okura, Setagaya, Tokyo 157-8535, Japan. ${ }^{2}$ Global Health Nursing, Faculty of Medicine, University of Tsukuba, 1-1-1, Tennodai, Tsukuba, Ibaraki 305-8577, Japan. ${ }^{3}$ Laboratory of Exercise Biochemistry and Neuroendocrinology, Faculty of Health and Sport Sciences, University of Tsukuba, Tsukuba 305-8574, Ibaraki, Japan. ${ }^{4}$ Division of Sport Neuroscience, Advanced Research Initiative for Human High Performacnce (ARIHHP), Faculty of Health and Sport Sciences, University of Tsukuba, Tsukuba 305-8574, Ibaraki, Japan. ${ }^{5}$ Mongolian National Institute of Physical Education, P.O.Box-224, Ikh Toiruu-49, Sukhbaatar district, Ulaanbaatar, Mongolia. ${ }^{6}$ Department of Neuropsychiatry, Graduate School of Medicine, The University of Tokyo, 7-3-1 Hongo, Bunkyo, Tokyo 113-8655, Japan. 'Division of Biostatistics, National Center for Child Health and Development, 2-10-1, Okura, Setagaya, Tokyo 157-8535, Japan.

Received: 3 December 2018 Accepted: 15 May 2019 Published online: 06 June 2019

\section{References}

1. World Health Organization. Global Status Report on Noncommunicable Diseases 2014. 2014.
2. Lee IM, Shiroma EJ, Lobelo F, Puska P, Blair S, Katzmarzyk P. Effect of physical inactivity on major non-communicable diseases worldwide: an analysis of burden of disease and life expectancy. Lancet. 2012;380(9838): 219-29.

3. World Health Organization. Global Strategy on Diet, Physical Activity and Health: Physical Activity and Young People.

4. Meeusen R, Meirleir KD. Exercise and brain neurotransmission. Sports Med. 1995;20:160-8.

5. Marmeleira J. An examination of the mechanisms underlying the effects of physical activiety on brain and cognition. Eur Rev Aging Phys Act. 2013;10:83-94.

6. Biddle SJH, Asare M. Physical activity and mental health in children and adolescents: a review of reviews. Br J Sports Med. 2011;45(11):886-95.

7. Harvey SB, Hotopf M, Overland S, Mykletun A. Physical activity and common mental disorders. Br J Psychiatry. 2010;197(5):357-64.

8. Watson A, Timperio A, Brown H, Best K, Hesketh KD. Effect of classroom-based physical activity interventions on academic and physical activity outcomes: a systematic review and meta-analysis. Int J Behav Nutr Phys Act. 2017;14(1):114

9. Howie EK, Pate RR. Physical activity and academic achievement in children: a historical perspective. J Sport Health Sci. 2012;1:160-9.

10. Sardinha LB, Marques A, Martins S, Palmeira A, Minderico C. Fitness, fatness, and academic performance in seventh-grade elementary school students. BMC Pediatr. 2014;14:176.

11. Ahn S, Fedewa AL. A meta-analysis of the relationship between children's physical activity and mental health. J Pediatr Psychol. 2011;36(4):385-97.

12. Donnelly JE, Hillman $\mathrm{CH}$, Castelli $\mathrm{D}$, Etnier JL, Lee $\mathrm{S}$, Tomporowski $\mathrm{P}$, et al. Physical activity, fitness, cognitive function, and academic achievement in children: a systematic review. Med Sci Sports Exerc. 2016;48(6):1197-222.

13. Have M, Nielsen JH, Gejl AK, Thomsen Ernst M, Fredens K, Stockel JT, et al. Rationale and design of a randomized controlled trial examining the effect of classroombased physical activity on math achievement. BMC Public Health. 2016;16:304.

14. Wright CM, Duquesnay PJ, Anzman-Frasca S, Chomitz VR, Chui K, Economos $C D$, et al. Study protocol: the fueling learning through exercise (FLEX) study - a randomized controlled trial of the impact of school-based physical activity programs on children's physical activity, cognitive function, and academic achievement. BMC Public Health. 2016;16(1):1078.

15. Smedegaard S, Christiansen LB, Lund-Cramer P, Bredahl T, Skovgaard T. Improving the well-being of children and youths: a randomized multicomponent, school-based, physical activity intervention. BMC Public Health. 2016;16(1):1127.

16. Hillman $\mathrm{CH}$, Castelli DM, Buck SM. Aerobic fitness and neurocognitive function in healthy preadolescent children. Med Sci Sports Exerc. 2005;37(11):1967-74.

17. Jager K, Schimidt M, Conzelmann A, Roebers CM. Cognitive and physiological effects of an acute physical activity intervention in elementary school children. Front Psychol. 2014;5:1473.

18. Verburgh L, Konigs M, Scherder EJ, Oosterlaan J. Physical exercise and executive functions in preadolescent children, adolescents and young adults: a meta-analysis. Br J Sports Med. 2014;48(12):973-9.

19. Kamijo K, Pontifex MB, Khan NA, Raine LB, Scudder MR, Drollette ES, et al. The negative Association of Childhood Obesity to cognitive control of action monitoring. Cereb Cortex. 2014:24:654-62.

20. Kamijo K, Pontifex MB, O'Leary KC, Scudder MR, Wu CT, Castelli DM, et al. The effects of an afterschool physical activity program on working memory in preadolescent children. Dev Sci. 2011;14(5):1046-58.

21. Moffitt TE, Arseneault L, Belsky D, Dickson N, Hancox RJ, Harrington H, et al. A gradient of childhood self-control predicts health, wealth and public safety. PNAS. 2011;108:2693-8.

22. Ekeland E, Heian F, Hagen KB. Can exercise improve self esteem in children and young people? A systematic review of randomised controlled trials. $\mathrm{Br} J$ Sports Med. 2005;39(11):792-8; discussion -8.

23. van der Niet AG, Smith J, Scherder EJA, Oosterlaan J, Hartman E, Visscher C. Associations between daily physical activity and executive functioning in primary school-aged children. J Sci Med Sport. 2015;18:673-7.

24. Castelli DM, Hillman CH, Buck SM, Erwin HE. Physical fitness and academic achievement in third- and fifth-grade students. J Sport Exerc Psychol. 2007.

25. Hyodo K, Dan I, Kyutoku Y, Suwabe K, Byun K, Ochi G, et al. The association between aerobic fitness and cognitive function in older men mediated by frontal lateralization. Neuroimage. 2016;125:291-300.

26. Suwabe K, Hyodo K, Byun K, Ochi G, Fukuie T, Shimizu T, et al. Aerobic fitness associates with mnemonic discrimination as a mediator of physical activity effects: evidence for memory flexibility in young adults. Sci Rep. 2017;7:5140.

27. Rasberry CN, Lee SM, Robin L, Laris BA, Russell LA, Coyle KK, et al. The association between school-based physical activity, including physical 
education, and academic performance: a systematic review of the literature. Prev Med. 2011;52(Supplement:S10-20.

28. Martin A, Booth J, Laird Y, Sproule J, Reilly J, Saunders D. Physical activity, diet and other behavioural interventions for improving cognition and school achievement in children and adolescents with obesity or overweight. Cochrane Database Syst Rev. 2018.

29. Ganchimeg U. Global school-based student health survey, Mongolia, 2013. 2013.

30. Job Table. Statistical Office of Ulaanbaatar. Ulaanbaatar. 2017 Available from: http://www.ubstat.mn/JobTables.aspx. Accessed 20 Mar 2018.

31. Alves CRR, Tessaro VH, Teixeira LA, Murakava $K$, Roschel $H$, Gualano B, et al. Influence of acute high-intensity aerobic interval exercise bout on selective attention and short-term memory tasks. Percept Mot Skills. 2014;118:63-72.

32. Gibala MJ, Little JP, MacDonald MJ, Hawley JA. Physiological adaptations to low-volume, high-intensity interval training in health and disease. J Physiol. 2012:590:1077-84

33. Kujach S, Byun K, Hyodo K, Suwabe K, Fukuie T, Laskowski R, et al. A transferable high-intensity intermittent exercise improves executive performance in association with dorsolateral prefrontal activation in young adults. Neuroimage. 2018;169:117-25.

34. Lambrick D, Stoner L, Grigg R, Faulkner J. Effects of continuous and intermittent exercise on executive function in children aged 8-10 years. Psychophysiology. 2016;53(9):1335-42.

35. Karageorghis Cl, Priest DL. Music in the exercise domain: a review and synthesis (part II). Int Rev Sport Exerc Psychol. 2012;5(1):67-84

36. Wininger SR, Pargman D. Assessment of factors associated with exercise enjoyment. J Music Ther. 2003:40:57-73.

37. Vanchindorj B, Naidansuren T, Bayartsogt B, Yerlan G, Narmandakh A, Batdelger $\mathrm{S}$, et al. Using the strengths and difficulties questionnaire (SDQ) to screen for child mental health status in Mongolia. Journal of Mental Disorders and Treatment. 2017;(01):03.

38. Youthinmind. Information for researchers and professionals about the Strengths \& Difficulties Questionnaires 2016 [Available from: http://www. sdqinfo.com. Accessed 20 Mar 2018

39. Diamond A. Executive functions. Annu Rev Psychol. 2013;64:135-68.

40. Heo M, Leon AC. Sample size requirements to detect an intervention by time interaction in longitudinal cluster randomized clinical trials. Stat Med. 2009;28(6):1017-27.

41. Borg G. Perceived exertion as an indicator of somatic stress. Scand J Rehabil Med. 1970;2(2):92-8

Ready to submit your research? Choose BMC and benefit from:

- fast, convenient online submission

- thorough peer review by experienced researchers in your field

- rapid publication on acceptance

- support for research data, including large and complex data types

- gold Open Access which fosters wider collaboration and increased citations

- maximum visibility for your research: over $100 \mathrm{M}$ website views per year

At BMC, research is always in progress.

Learn more biomedcentral.com/submissions 